\title{
Statistical Feature based Activity Classification
}

\author{
Supriya Shete \\ Dept. of Instrument and Control \\ Cummins College of Engineering Pune, India
}

\author{
Revathi Shriram \\ Dept. of Instrument and Control \\ Cummins College of Engineering Pune, India
}

\begin{abstract}
Activity refers two activities and in this work to classification between two activities is achieved with the help of statistical feature extraction technique. [5] The term silent activity refers to the two processes in which we are proposing a method to predict Cognitive and Non cognitive tasks performed by the human brain. Electroencephalogram (EEG) is the electrical signal of brain which contains valuable information. In this work EEG and its frequency sub-bands have been analyzed to detect silent activity signal. The electroencephogram (EEG) contains information about brain hence the sub band decomposition of EEG is used for analyzing many brain diseases. [1] The subband decomposition means to extract various brain waves with different frequency bands such as alpha, beta, delta, theta and gamma from EEG signal to get more information from it. The work was carried out to extract various brain waves using discrete wavelet transform. The EEG signal is decompose into five sub bands alpha, beta, gamma, theta, and delta. [2] A wavelet transform has been applied to decompose the EEG into its sub bands. Statistical features Standard deviation, Covariance is calculated for each sub-band. The effective classification of EEG used for brain computer interface and can be used for silent communication or for recognizing different mental tasks. [5]
\end{abstract}

\section{Keywords}

Sub-band Decomposition, EEG, Wavelet, Statistical features, Standard Deviation, Variance

\section{INTRODUCTION}

In this work silent activity refers that activities which performs without any audible sound or any muscle movement.Cognitive task include any simple mathematical operation and Non cognitive task include a sample speech. [10] A subject is supposed to perform these two tasks in silent fashion means without moving any articulator muscle or producing any audible sound. Task had to be marked in a procedure controlled by the subject. Each part of the brain has a specific, important function, often a profoundly important function, and each part contributes to the healthy functioning of our body. [12]

EEG is conventionally described consisting of patterns within four frequency bands, or brain waves named Delta $(<4 \mathrm{~Hz})$, Theta $(4-7 \mathrm{~Hz})$, Alpha $(8-12 \mathrm{~Hz})$, Beta $(13-35 \mathrm{~Hz})$. [9]

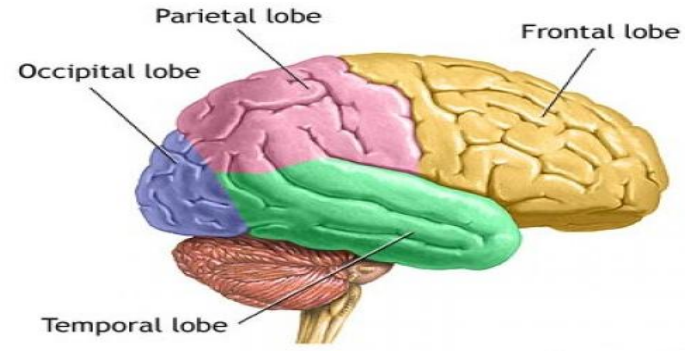

Figure: 1Brain Lobes [1]
To measure the EEG extra cranially, electrodes are attached to the scalp with a conducting gel. Each electrode conducts the potential in the surrounding head area to an assigned channel of an amplifier where it is usually exposed to a low pass filter prior amplification. [7] In most modern settings the signal is appropriately sampled and the digitized data is then forwarded to a computer for further processing depending on the individual application. [8]

\section{CHANNELS USED FOR BOTH ACTIVITY}

The 10-20 system or International $\quad 10-20$ system is an internationally recognized method to describe and apply the location of scalp electrodes. Frontal Lobe is one of the four lobes of the cerebral hemisphere. It controls attention, behavior, abstract thinking, problem solving, creative thought, emotion, intellect, initiative, judgment, coordinated movements, muscle movements, smell, physical reactions, and personality.

Parietal Lobe is one of the four lobes of the cerebral hemisphere. It controls tactile sensation, response to internal stimuli, sensory comprehension, some language, reading, and some visual functions. [1][4]

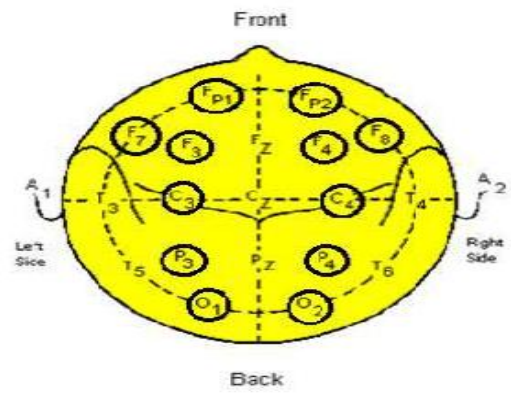

Figure 2: Electrodes used for Cognitive activity [1]

The subject was shown images consisting number of questions one by one. Each question will be display for 7-8 sec and then the next question comes and so on. The subject was asked to mentally compose a answer to that question without vocalizing or making any physical movements. The three lobes are used for non-cognitive activity are frontal lobe, temporal lobe and occipital lobe. The function of each lobe is as follows: [1]

Frontal Lobe is one of the four lobes of the cerebral hemisphere. It controls attention, behavior, abstract thinking, problem solving, creative thought, emotion, intellect, initiative, judgment, coordinated movements, muscle movements, smell, physical reactions, and personality. [4]

Parietal Lobe is one of the four lobes of the cerebral hemisphere. It controls tactile sensation, response to internal stimuli, sensory comprehension, some language, reading, and some visual functions.[1]

Temporal lobe is one of the four lobes of the cerebral hemisphere of the cerebral hemisphere. It controls auditory and 
visual memories, language, some hearing and speech, language, plus some behavior. [1]

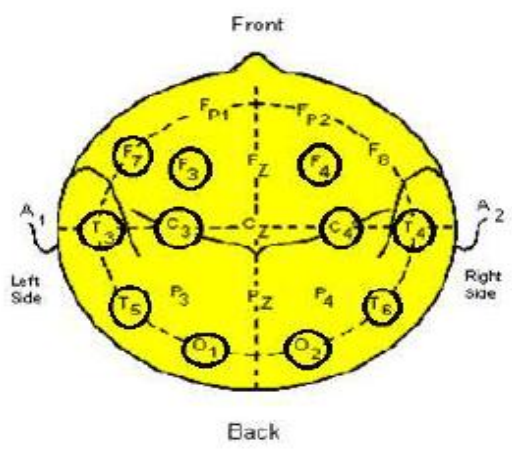

Figure 3: Electrodes for Noncognitive activity [1]

\section{BLOCK DIAGRAM OF SYSTEM}

The .eeg file is converted into text file or excel file with the help of EEG analysis software. In the EEG analysis software load .eeg file and go to tool and convert it into text or excel file. I am converting the .eeg file into excel sheet because it is easy for me to ploy the particular channel in to the Matlab. After applying wavelet transform to each channel we get five brain wave (gamma, alpha, beta, theta, and delta). The figure 3 shows the block diagram of the system. The feature extraction techniques used are Standard deviation and variance. With the help of these techniques the standard deviation and variance values are to be calculated. This range is given to the classifier, which is my studied database. [4] When the activity is to be performed then classifier compares the output to the studied database and classifier classifies the activity is Cognitive or Noncognitive. [2]

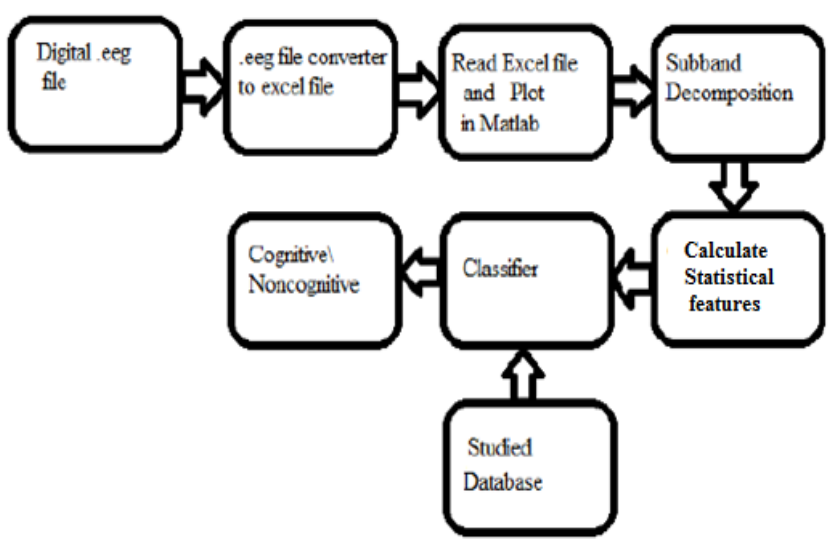

Figure 4: Block Diagram of Statistical Features based Activity Classification

\section{PPT FOR COGNITIVE AND FOR NON- COGNITIVE TASK}

Silent tasks are performed by persons so some steps has followed first ask subject for relaxation then the first image will be displayed. Solve the problem or answer to the question in silent manner then second image will be displayed and so on. And capture the EEG when person performs the tasks.

\subsection{For Cognitive Activity}

The subject was shown images consisting of nontrivial multiplication problems, such as 79 plus 54 , and was asked to solve them without vocalizing or making any physical movements. Means without any audible sound or muscle movement person solves these problems. Figure 5shows PPT for Noncognitive activity

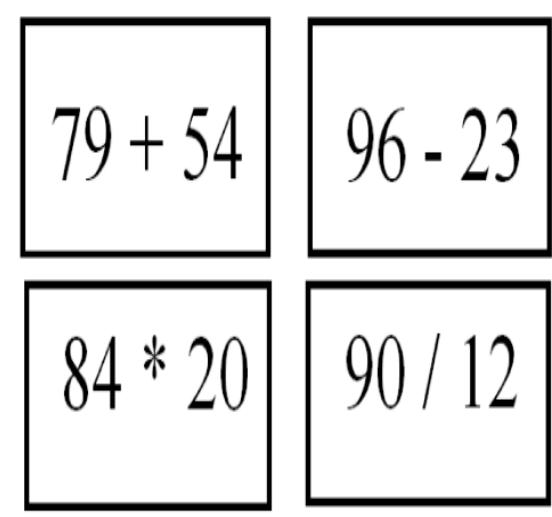

Figure 5: PPT for Cognitive activity

\subsection{For Non-cognitive Activity}

The subject was shown images consisting number of questions one by one. Each question will be display for $4 \mathrm{sec}$ and then the next question comes and so on. The subject was asked to mentally compose an answer to that question without vocalizing or making any physical movements. Means person has to answer those questions in a silent manner.

Figure 6 shows PPT for Noncognitive activity

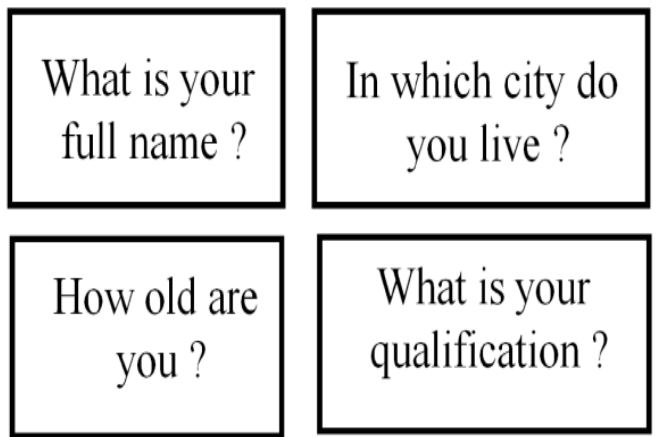

Figure 6: PPT for Noncognitive activity 


\section{FLOWCHART FOR FEATURE EXTRACTION TECHNIQUES}

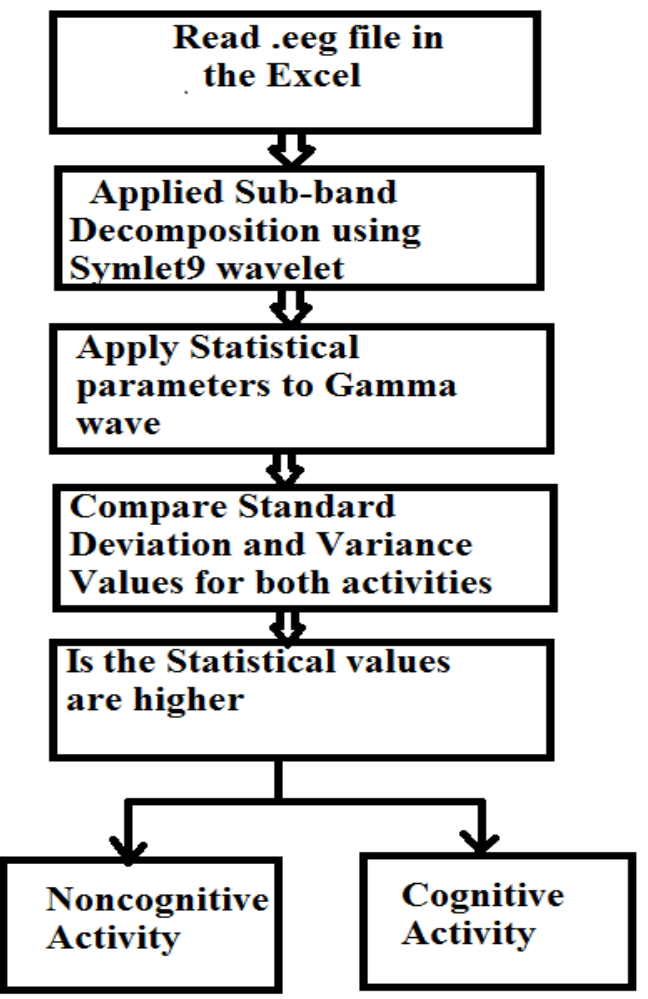

The flowchart shows how the feature extraction techniques statistical parameters are used.

Here the statistical parameters used are standard deviation and variance.

\subsection{Standard Deviation}

Standard deviation measures how the numbers are spread out the standard deviation shows how much variation or dispersion from the average exists. [5] A low standard deviation indicates that the data points tend to be very close to the mean high standard deviation indicates that the data points are spread out over a large range of values. The standard deviation of a random variable is the square root of its variance. [6]

Here the operator $E$ denotes the average or expected value of $X$. Then the standard deviation of $X$ is

$$
\sigma=\sqrt{\mathrm{E}\left[(X-\mu)^{2}\right]}
$$

\subsection{Variance}

A measurement of the spread between numbers in a data set. The variance measures how far each number in the set is from the mean. [5] Variance is calculated by taking the differences between each number in the set and the mean, squaring the differences (to make them positive) and dividing the sum of the squares by the number of values in the set.[6] The variance of a random variable $X$ is its second central moment, the expected value of the squared deviation from the mean $\mu=\mathrm{E}[X]$

$$
\operatorname{Var}(X)=\mathrm{E}\left[(X-\mu)^{2}\right] .
$$

\section{RESULT}

Table 1 shows the comparison of standard deviation values for gamma between Cognitive and Non-cognitive activity. Channel taken for cognitive activity is F4-C4 and for Noncognitive activity is T3-T5. The comparison is done with the help of database of seven subjects. The database is taken when subject performing these two activities in silent manner. Then the subband decomposition is applied to that channels we get gamma for cognitive activity and gamma for Noncognitive activity. The table 1 shows that the standard deviation values for Noncognitive activity is more as compared to cognitive activity. So with the help of statistical parameter (standard deviation) is useful for classification between two activities.

Table 1: Standard Deviation values for cognitive and Noncognitive tasks

\begin{tabular}{|l|l|l|}
\hline $\begin{array}{l}\text { Standard } \\
\text { Deviation Values }\end{array}$ & $\begin{array}{l}\text { Camma ofCognitive Activity } \\
\text { (Channel F4-CA) }\end{array}$ & $\begin{array}{l}\text { Grmma of Non-cognitive } \\
\text { Activity (Channel T3 - T5) }\end{array}$ \\
\hline Subject 1 & 23.3 & 39.03 \\
\hline Subject2 & 22.13 & 43.65 \\
\hline Subject 3 & 24.5 & 42.25 \\
\hline Subject 4 & 21.5 & 49.88 \\
\hline Subject 5 & 23.03 & 35.76 \\
\hline Subject 6 & 24.7 & 27.3 \\
\hline Subject7 & 23.9 & 32 \\
\hline
\end{tabular}

Table 2 shows the comparison of variance values for gamma between Cognitive and Non-cognitive activity. Channel taken for cognitive activity is F4-C4 and for Noncognitive activity is T3-T5. The comparison is done with the help of database of seven subjects. The table 2 shows that the variance values for Noncognitive activity is more as compared to cognitive activity. So with the help of statistical parameter (variance) is useful for classification between two activities.

Table 2: Variance values for cognitive and Noncognitive tasks

\begin{tabular}{|l|l|l|}
\hline Variance Values & $\begin{array}{l}\text { Gamma of Cognitive } \\
\text { (Channel F4-C4) }\end{array}$ & $\begin{array}{l}\text { Gamma of Non-cognitive } \\
(\text { Channel T3-T5) }\end{array}$ \\
\hline Subject 1 & 546.1 & 1500 \\
\hline Subject 2 & 490.1 & 1900 \\
\hline Subject 3 & 604.19 & 1700 \\
\hline Subject 4 & 462.3 & 2400 \\
\hline Subject 5 & 530.4 & 1200 \\
\hline Subject 6 & 614.3 & 749.9 \\
\hline Subject 7 & 575.19 & 1002 \\
\hline
\end{tabular}

\section{DISCUSSION}

In this work two activities are referred which performs in silent manner first is cognitive activity where a mathematical calculation is performed and second is Noncognitive activity where questions are given to the subject. We are proposed a 
method to predict cognitive and Noncognitive tasks performed by the human brain using Statistical parameter analysis signals. For seven subjects' statistical features (means standard deviation and variance) values of Noncognitive activity is more as compare to cognitive activity for gamma wave. This can be used as a mode of communication or wheelchair control for paralyzed patients and also in EEG biofeedback systems.

\section{CONCLUSION}

Cognitive and Non cognitive tasks performed by the human brain. Activity Recognition based on EEG signal is very useful, safe, noninvasive procedure. We are proposing a method to classify these two activities. With the help of all brain lobes and its function we refer six channels for cognitive activity and seven channels for Noncognitive activity. Our result shows that the discrimination between cognitive and Noncognitive activity is achieved with the help of statistical parameters. For seven subjects statistical features values (standard deviation and variance)of Non-cognitive activity is more as compare to Cognitive activity for Gamma wave and for Beta wave also.

\section{ACKNOWLEDGMENT}

I would like to express my deep and sincere gratitude to my principal Dr. Madhuri Khambete Madam, and head of the department Dr. Ashok Gaikwad sir and professors of Instrumentation department of Cummins College of engineering provided extensive comments and suggestions on the work. Thanks to all of them.

\section{REFERENCES}

[1] Anatomy of Brain, Brain parts and function of each part, Encyclopedia

[2] 'EEG Signal Analysis for Silent Visual Reading Classification' Oliveira, O. Grigori, and N. Guimarães. International Journal of Circuits, Systems and Signal processing

[3] "Classification of EEG Signals under Different Mental Tasks Using Wavelet Transform and Neural Network with
One Step Secant Algorithm", Dipti Upadhyay Department of Applied Mechanics, Motilal Nehru National Institute of Technology, Allahabad, India

[4] Discriminating Mental States Using EEG Represented by Power Spectral Density, Jack Culpepper, Department of Computer Science, Harvey Mudd College, Claremont

[5] Feature selection for Epilepsy Detection using EEG Mandeep Singh and Sunpreet Kaur International Journal of Information Technology and Knowledge Management December 2012

[6] Chai Tong Yuen, Woo san san, Classification of Human Emotions form EEG signal using Statistical Features and Neural Network, International Journal of Integrated Engineering

[7] Norizam Sulaiman, Novel Methods for Stress Features Identification using EEG Signals,Faculty of Electrical and Electronics Engineering University Malaysia Pahang , Malaysia

[8] John Musson, Jiang Li, (2002) A Comparative Survey of PSD Estimation Methods for EEG Signal Analysis,IEEE Signal Processing Magazine Old Dominion University, Norfolk

[9] R. Palaniappan and P. Raveendran(2001) Cognitive Task Prediction using Parametric analysis Malaysian Journal of Computer Science.

[10] Tian Lan, Andre Adami,(2006),Estimating Cognitive state using EEG signal. IEEE Trans Rehabil Eng.

[11] Porbadnigk, A. (2008). EEG-based speech recognition: Impact of experimental design on performance. Institute Algorithmen und Kognitive System.

[12] Wester, M. (2006). Unspoken speech speech recognition based on electroencephalography. Master's thesis, Institute Theoretische Informatik University 